\title{
Impact of Early Palliative Care on Survival in Advanced-stage Cancer Patients: An Institution-based Retrospective Cross- sectional Study
}

\author{
Niketa Thakur, Sushmita Ghoshal
}

Post Graduate Institute of Medical Education and Research, Chandigarh, India.

\begin{abstract}
Background: While treating patients with the advanced disease it becomes very challenging for the clinicians to decide as to when the active treatment is justified and when should palliative care be introduced in patient's treatment. American Society of Clinical Oncology (ASCO) suggested that patients with advanced cancer, regardless of patient or outpatient, should receive dedicated palliative care services, early in the disease course, simultaneous with active treatment. Similar recommendations have been proposed by the European Society of Medical Oncology (ESMO). Keeping in view this background, we aimed to do this study. Aim: To study the impact of the introduction of early palliative care into routine oncological management on patient's survival. Methods: This retrospective cross-sectional study is based on hospital data for patients who died in the hospice between the year 2017 and 2018. The complete information including age, gender, date of death, diagnosis, procedures, and medical interventions, as well as the date of documentation, was obtained of each patient. Results: A total of 27 patients were enrolled in this study. The mean age at presentation was 50.3 years. $11(40.7 \%)$ patients received oncological treatment integrated with palliative care in the last month of life. The median duration of survival from diagnosis till death was 7 months in the patients who received only palliative care and 10 months in patients who received integrated onco-palliative care in the last month of life $(\mathrm{P}=0.452) .14(51.8 \%)$ patients received early palliative care. The median survival of the patients who received early palliative care and of those who were offered palliative care towards the end of life was 8.5 months and 7 months respectively $(\mathrm{P}=0.040)$. Conclusion: Based on our results, we strongly believe that early palliative care when integrated into standard oncological treatment in advance stage cancer patients can prolong patient's survival.
\end{abstract}

Keywords: Early palliative care- advanced cancer- end of life- hospice- death

Asian Pac J Cancer Care, 4 (4), 151-155

\section{Introduction}

According to WHO estimates for 2011, cancer is a leading cause of death worldwide. The worldwide incidence of malignancy is on the rise with a related increase in the number of patients living with disabilities because of their primary illness as well as its treatment complications. In everyday clinical practice while treating patients with the advanced disease it turns out to be extremely difficult for the clinicians to choose about when the active treatment is advocated in the last days of life and when should palliative care be integrated into patient's treatment. It is not an easy choice and the circumstances are made progressively complex when patients demand
Submission Date: 04/02/2019 Acceptance Date: 07/20/2019

serious treatment measures notwithstanding when the prognosis is dismal [1-2]. A definitive objective of palliative care is to improve quality of life for both the patient and the family, regardless of diagnosis.

Earle et al. characterized specific clinical quality indicators for overtreatment, incorrect treatment, and undertreatment during the end of the life [3]. The indicators distinguished were: 1 ) receiving chemotherapy in the last 14 days of life and/or start of chemotherapy during the last 30 days of life (overtreatment); 2) more than one emergency hospital admission and/or intensive-care unit admission during the last month of life (incorrect treatment); 3) involvement of hospice and/or specialized palliative services less than 3 days before death

Corresponding Author:

Dr. Niketa Thakur

Post Graduate Institute of Medical Education and Research, Chandigarh, India.

Email: niketathakur7@gmail.com 
(undertreatment) [4].

American Society of Clinical Oncology (ASCO) suggested that patients with advanced cancer should receive dedicated palliative care services, early in the disease course, simultaneous with active treatment. Referring patients to interdisciplinary palliative care teams is optimal [5].

Similar recommendations have been proposed by the European Society of Medical Oncology (ESMO) [6]. The time point at which palliative care should be included were published in a consensus-based German guideline on palliative medicine for patients with incurable cancer [7]: 1) all patients with malignancy should receive information about palliative care, independent of the disease stage; 2) following diagnosis of incurable cancer, all patients should be offered palliative care, independently of whether tumor-specific treatment is being administered; 3) specialist palliative care should be integrated into oncological decision-making processes. Keeping in view this background, we aimed to do this study.

\section{Methods}

Aim

To study the impact of early introduction of palliative care into routine oncological management on the patient's survival.

\section{Study design}

This retrospective analysis is a cross-sectional study based on hospital data for the advanced stage cancer patients who died in the hospice of Post Graduate Institute of Medical Education and Research, Chandigarh, India, between April 1st, 2017 and March 31st, 2018 $(n=27)$. Over for 1 year, 34 patients died in a hospice. Out of these, 27 patients had presented with advanced-stage cancer at our outpatient clinic. The study protocol was approved by the Institutional Ethics Committee. The study population was solely related to deceased patients with a diagnosis of cancer following version 10 of the International Classification of Diseases (ICD-10). All the patients had a solid tumor.

The palliative care team at our institute comprises of physicians, palliative care nurses and medical social workers. The team provides specialist palliative care for patients with advanced disease and in the terminal phase. The services incorporate continuous palliative consultation and collaborative treatment in patients with complex manifestations and requirements.

The patient data was obtained from the hospital electronic medical records. We had complete access to information including age, gender, primary cancer, stage, performance status, date of death, diagnosis, procedures, medical interventions and the start of palliative care. The information regarding the frequency of admission, chemotherapy during the last 30 days, invasive procedures, palliative radiotherapy was also obtained. We compared the survival between early and late palliative care referral. Early palliative care was defined as palliative care referral within 8 to 12 weeks of the start of routine oncological treatment.

\section{Statistical analyses}

The frequencies relating to clinical characteristics, medical procedures, interventions, and contact with the hospital palliative care team were presented in absolute and percentage figures. The chi-squared test was used for categorical data. The significance level was set at $\mathrm{p}<0.05$. The analyses were carried out using the IBM SPSS Statistics program, version 23 (SPSS Inc., Chicago, Illinois).

\section{Results}

A total of 27 patients were enrolled. The characteristics of the deceased patients are shown in Table number 1. All of these patients had a solid tumor. There were more females $(59.1 \%)$ than males $(48.1 \%)$. The mean age at presentation was 50.3 years. The intent of treatment at presentation was radical in $29.6 \%$ while the intent was palliative in $70.4 \%$ of the patients.

$11(40.7 \%)$ patients received integrated onco-palliative care in the last month of life. Out of these, 4 (14.8\%) patients received active chemotherapy in the last month of life. Out of 4, 2 had gastrointestinal malignancy and the other two had oligodendroglioma grade 3 and renal cell carcinoma respectively. The intent of chemotherapy was

Table 1. Patient Characteristics

\begin{tabular}{lc}
\hline \multicolumn{2}{l}{ Number of patients $(\mathrm{n}=27)(\%)$} \\
\hline Gender & $13(48.1)$ \\
Male & $14(51.9)$ \\
Female & \\
Age & $6(22.2)$ \\
$<40$ & $13(48.1)$ \\
$40-59$ & $8(29.6)$ \\
$60-79$ & 0 \\
$>80$ & \\
Solid Tumors & $6(22.2)$ \\
GIT & $5(18.5)$ \\
Head and Neck & $4(14.8)$ \\
Gynaecological & $4(14.8)$ \\
Breast & $4(14.8)$ \\
Genitourinary & $1(3.7)$ \\
CNS & $2(7.4)$ \\
Sarcoma & $1(3.7)$ \\
Others & \\
Intent of treatment at presentation & $14(51.9)$ \\
Radical & $13(48.1)$ \\
Palliative & \\
Duration between diagnosis and cancer death \\
$<6$ months \\
6-12 months & $10(37.0)$ \\
$>12$ months & $9(33.3)$ \\
& \\
&
\end{tabular}




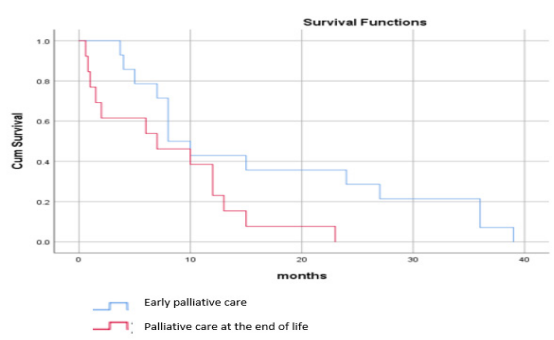

Figure 1. Overall Survival Early Palliative Care Versus Palliative at the End of Life $(\mathrm{P}=0.04)$

palliative in all the patients. In two patients, chemotherapy was second line while in the other two it was first line. One patient developed skin complications viz bullous impetigo and contact dermatitis. This patient had to be put on injectable antibiotics. Integrated onco-palliative care comprised of palliative care integrated with standard oncological treatment which is either palliative radiotherapy or palliative chemotherapy. The median duration of survival from diagnosis till death was 7 months in the patients who received only palliative care and 10 months in patients who received integrated oncopalliative care in the last month of life $(\mathrm{P}=0.452)$. There was slight survival benefit with palliative care integrated with routine oncological treatment in the last month of life but the patients who received active chemotherapy had more adverse events related to chemotherapyinduced toxicity. The use of chemotherapy in patients in the terminal phase of their illness should involve a very cautious approach. However, patients who received palliative radiotherapy had good symptom control.

None of the patients underwent resuscitation at the end of life as the consent for "do not resuscitate" was taken of every patient in the hospice. 2 patients $(7.4 \%)$ received blood transfusions in the last week of life.

About diagnostic measures at the end of life (last week/month), diagnostic imaging such as CT and MRI were carried out in $22.2 \%$ of the patients.

None of the patients had surgery in the last 4 weeks of life. The frequencies of medical interventions in last week and last month of life are mentioned in Table number 2 .

Contact with the palliative care team had been established in all the patients in the last 4 weeks of life. The duration of care of the hospital palliative care team at the time of death was most often between 2 to 5 days. The duration between the first palliative care referral and

Table 2. Frequencies of Medical Interventions in Last Week and Last Month of Life

\begin{tabular}{lcc}
\hline & Last week of life & Last month of life \\
\hline Chemotherapy & $1(3.7 \%)$ & $4(14.81 \%)$ \\
Radiotherapy & $3(11.1 \%)$ & $7(25.9 \%)$ \\
Surgery & 0 & 0 \\
ICU admission & 0 & 0 \\
Imaging (MRI/CT) & 0 & $6(22.2 \%)$ \\
Blood transfusion & 0 & $2(7.4 \%)$ \\
\hline
\end{tabular}

Table 3. Duration between the First Palliative Care Referral and Patient's Death

\begin{tabular}{lc}
\hline Duration & No. of patients $(\%)$ \\
\hline 1 -10days & $2(7.4)$ \\
$11-20$ days & $4(16.6)$ \\
$21-30$ days & $2(7.4)$ \\
$>1$ month-3 months & $4(14.8)$ \\
$>3$ months-6 months & $12(44.4)$ \\
$>6$ months-12 months & $2(7.4)$ \\
$>12$ months & $1(3.7)$ \\
\hline
\end{tabular}

the patient's death is mentioned in Table number 3 .

Early palliative care was defined as palliative care referral within 8 to 12 weeks of the start of routine oncological treatment. $14(51.8 \%)$ patients received early palliative care. The median survival of the patients who received early palliative care and of those who were offered palliative care towards the end of life was 8.5 months and 7 months respectively $(\mathrm{P}=0.040)$ (Figure 1). The patients in whom the palliative care was commenced in the early phase had better symptom control than those who were given palliative care in the last days of life.

The median duration of stay in hospice among patients who received early palliative care was 10 days as compared to 5 days in patients who received palliative care in the terminal phase. The patients with a longer stay at the hospice or having frequent hospice admissions were found to have a better understanding of issues relating to life and spirituality. Hospice care helped ease the anxiety of both the patients and family members.

\section{Discussion}

This study depicts the trends and patterns of care provided to advance stage cancer patients who spent their final phase of life in a hospice and died there. In our study, we find slight improvement in the patient's survival in whom the palliative care was introduced at an early phase of oncological treatment.

The role of chemotherapy during the last days of life is usually palliative. Its life-prolonging effect is usually slight, and there is a risk of worsening of the patient's quality of life due to treatment-related toxicities. For this reason, a cautious approach is needed when applying chemotherapy to the patients in their terminal phase of the illness.

In a study, among patients with very advanced tumor stages who received palliative chemotherapy, patients with good performance status (ECOG score 1) had a poorer quality of life with treatment, while in patients with a moderate or poor functional status (ECOG score $2 \pm 3$ ), quality of life was unchanged. Palliative chemotherapy might add to an improvement in the quality of life [8-9] or reduce symptoms but cause adverse effects, prevent the patient from engaging in meaningful review of the life and preparing for death and preclude the entry into hospice [10].

In our study, chemotherapy had been administered 
within the last 4 weeks of life in 4 cancer patients $(14.8 \%)$ and out of these, one patient $(3.7 \%)$ received chemotherapy in the last two weeks of life. The intent of chemotherapy was palliative in all these patients. The slight life-prolonging benefit was seen but these patients had more adverse events in the form of chemotherapy-induced toxicities. Excessive palliative chemotherapy has toxic effects that may result in less time for appropriate hospice management for end-stage cancer patients. The potential benefits and side effects of palliative chemotherapy should be explained to the patients and caregivers in detail, and decisions should be mutually agreed upon.

The various palliative modalities such as palliative chemotherapy and palliative radiotherapy can improve the symptoms of end-stage cancer patients and hence may improve their quality of life. Palliative treatment courses of 8 to 30 Gy given in 1 to 10 fractions are useful for a wide range of scenarios such as acute pain or bleeding due to metastatic lesions. Similarly, in our study, patients who received palliative radiotherapy as a part of routine oncologic treatment had better symptom outcome.

Patients with malignant diseases often suffer from physical symptoms and unpleasant psychosocial circumstances not only in very advanced situations or in their terminal phase of life, but also earlier in the disease course. The need for early integration of palliative care into the routine treatment of cancer patients has been upheld in various studies [11-20]. In a study by Hui et al [16], early specialist palliative care improved end-of-life care outcomes. Early integration of palliative care leads to reduced burden of physical and mental symptoms, fewer hospital admissions, larger numbers of transfers to a hospice, longer hospice stays, and fewer aggressive treatment procedures at the end of life. As per the German Palliative Care Guidelines, palliative care should be integrated at the diagnoses of the incurability of an oncological disease [7].

Jennifer S. Temel [21] and colleagues showed that among patients with metastatic non-small cell lung cancer, early palliative care led to significant improvements in both qualities of life and state of mind. As compared with patients receiving standard care, patients receiving early palliative care had longer survival (11.6 months vs. 8.9 months, $\mathrm{p}=0.02$ ). Patients receiving palliative care received significantly fewer active oncological treatments than other patients $(33 \%$ vs. $54 \%$; $p=0.05)$. Similarly, in our study also, we find prolonged survival among patients who received early palliative care.

Studies have shown that hospice is associated with a better quality of life (QoL) near death, [22] and also reductions in bereaved caregivers' risk of developing major depressive disorder [23]. The American Society of Clinical Oncology and other major professional societies have long recommended hospice as the best available care for dying patients [24]. Evidence suggests that palliative or hospice care integrated with routine oncology care improves health outcomes. Project Safe Conduct was started to integrate hospice care into lung cancer care at the Ireland Cancer Center. Before the study, $13 \%$ of patients with advanced lung cancer were referred to hospice; afterward, $80 \%$ of such patients enrolled in hospices and the average length of stay in hospice increased from 10 days to 44 days [25]. The one randomized trial of concurrent hospice care plus usual oncology care vs usual oncology care alone showed that the group with concurrent care lived slightly longer (not statistically significant), had a quality of life preserved longer, used less chemotherapy, and transitioned to hospice enrollment sooner [26]. In our study, all the patients spent the last few days of their life in a hospice. Patients who received early palliative care had a longer median duration of stay in a hospice and had prolonged survival.

The patient's preferred location for receiving end-of-life care should be determined. Most patients with cancer would prefer to spend one's remaining time at home but sometimes their care needs or support system at home cannot support their care. Some patients or their attendants request to receive end-of-life care in a skilled nursing facility or hospice. A prospective study showed that patients dying in an ICU had higher levels of physical and emotional distress compared with patients dying at home or in hospice. Caregivers of these patients had a greater incidence of grief disorder [27]. In our study, none of the patients had an intensive care unit admission in the last month of life.

Another important aspect that we come across with increased utilization of hospice and palliative care services is a significant reduction in the expenditure of medical treatment [28]. Possible reasons for cost savings can be attributed to a reduction in the use of medical services, overall hospital costs, laboratory and intensive care unit costs, and a significant decrease in hospital admissions, nursing home admissions, emergency department visits, and use of outpatient consultation services.

In our study, we studied the impact of early introduction of palliative care into routine oncological management on the patient's survival. We found a survival advantage among patients who received early palliative care along with routine oncological care. Palliative care neither hastens death nor prolongs life rather celebrates life and allows individuals to live well until a natural end.

There are several limitations of this study. As it is a retrospective study done in a single institution over a short period, it is difficult to generalize the results of the study. The study was limited to deceased cancer patients who died only in the hospice. The sample size was relatively small.

In conclusions, based on our results, we strongly believe that early palliative care when integrated into standard oncological treatment in advance stage cancer patients can prolong patient's survival. Integrated palliative care might help in reducing the level of potential overtreatment and improving patient outcomes, including symptom burden. However, as the sample size was very small, large scale studies need to be done to validate the results. 


\section{References}

1. Chu DT, Kim SW, Hsu HK, Cok G, Roubec J, Patil S, et al. Patient attitude towards chemotherapy and survival: a prospective observational study in advanced non-small cell

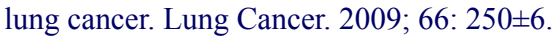

2. Slevin ML, Stubbs L, Plant HJ, Wilson P, Gregory WM, Armes PJ, et al. Attitudes to chemotherapy: comparing views of patients with cancer with those of doctors, nurses and general public. BMJ. 1990; 300(6737): 1458 \pm 60 .

3. Earl CC, Park ER, Lai B, Weeks JC, Ayanian JZ, Block S. Identifying potential indicators of the quality of end-of-life cancer care from administrative data. J Clin Oncol. 2003; 21(6): $1133 \pm 8$.

4. Earl CC, Neville BA, Landrum MB, Souza JM, Weeks JC, Block SD, et al. Evaluating claims-based indicators of the intensity of end-of-life cancer care. Int J Qual Health Care. 2005; 17(6): 505 \pm .

5. American Society of Clinical Oncology (ASCO): Integration of Palliative Care Into Standard Oncology Care. American Society of Clinical Oncology Clinical Practice Guideline Update.

6. Cherny N, Catane R, Schrijvers D, Koke M, Strasser F. European Society for Medical Oncology (ESMO). Program for the integration of oncology and Palliative Care: A 5-year review of the Designated Centers' incentive program. Ann Oncol. 2010; 21: 362 \pm 9.

7. Arbeitsgemeinschaft der Medizinisch-Wissenschaftlichen Fachgesellschaften \pm AWMF. Leitlinienprogramm Onkologie (Deutsche Krebsgesellschaft, Deutsche Krebshilfe, AWMF). Palliativmedizin fǜ $r$ Patienten mit einer nicht heilbaren Krebserkrankung. Langversion 1.0, 2015: AWMFRegisternummer: 128/001OL;

8. Swetz KM, Smith TJ. Palliative chemotherapy: when is it worth it and when is it not? Cancer J. 2010; 16(5): 467 \pm 72 .

9. Harrington SE, Smith TJ. The role of chemotherapy at the end of life: when is enough, enough? JAMA. 2008; 299(22): $2667 \pm 78$.

10. Prigerson HG, Bao Y, Shah MA, Paulk ME, LeBlanc TW, Schneider BJ, et al. Chemotherapy Use, Performance Status, and Quality of Life at the End of Life. JAMA Oncol. 2015; 1(6): $778 \pm 84$.

11. Davis MP, Temel JS, Balboni T, Glare P. A review of the trials which examine early integration of outpatient and home palliative care for patients with serious illnesses. Ann Palliat Med. 2015; 4(3): 99 \pm 121

12. Zhi WI, Smith TJ. Early integration of palliative care into oncology: evidence, challenges and barriers. Ann Palliat Med. 2015; 4(3): 122 \pm 31 .

13. Hui D, Bruera E. Models of integration of oncology and palliative care. Ann Palliat Med. 2015; 4(3): 89 \pm 98 .

14. Bakitas MA, Tosteson TD, Li Z, Lyons KD, Hull JG, Li Z, et al. Early versus delayed initiation of concurrent palliative oncology care: patient outcomes in the ENABLE III randomized controlled trial. J Clin Oncol. 2015; 33(13): $1438 \pm 45$.

15. Dalgaard KM, Bergenholtz H, Espenhain Nielsen M, Timm H. Early integration of palliative care in hospitals: A systematic review on methods, barriers, and outcome. Palliat

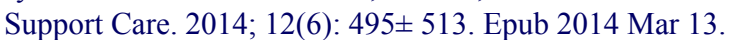

16. Zimmermann C, Swami N, Krzyzanowska M, Hannon B, Leighl N, Oza A, et al. Early palliative care for patients with advanced cancer: a cluster-randomised controlled trial. Lancet. 2014; 383(9930): 1721 \pm 30.

17. Greer JA, Pirl WF, Jackson VA, Muzikansky A, Lennes IT, Heist RS, et al. Effect of early palliative care on chemotherapy use and end-of-life care in patients with metastatic non-small-cell lung cancer. J Clin Oncol. 2012; 30(4): $394 \pm 400$.

18. Temel JS, Greer JA, Admane S, Gallagher ER, Jackson VA, Lynch TJ, et al. Longitudinal perceptions of prognosis and goals of therapy in patients with metastatic non-small-cell lung cancer: results of a randomized study of early palliative care. J Clin Oncol. 2011; 29(17): 2319 \pm 26.

19. Pantilat SZ, O'Riordan DL, Dibble SL, Landefeld CS. Hospital-based palliative medicine consultation: A randomized controlled trial. Arch Intern Med. 2010; 170(22): $2038 \pm 40$

20. Gade G, Venohr I, Conner D, McGrady K, Beane J, Richardson RH, et al. Impact of an inpatient palliative care team: a randomized control trial. J Palliat Med. 2008; 11(2): $180 \pm 190$.

21. Temel JS, Greer JA, Muzikansky A, Gallagher ER, Admane $\mathrm{S}$, Jackson VA, et al. Early palliative care for patients with metastatic non-small-cell lung cancer. N Engl J Med. 2010; 363(8): $733 \pm 742$

22. Teno JM, Clarridge BR, Casey V, et al. Family perspectives on end-of-life care at the last place of care. JAMA. 2004;291(1):88-93.

23. Kris AE, Cherlin EJ, Prigerson H, et al. Length of hospice enrollment and subsequent depression in family caregivers: 13-month follow-up study. Am J Geriatr Psychiatry. 2006;14(3):264-9.

24. Smith TJ, Schnipper LJ. The American Society of Clinical Oncology program to improve end-of-life care. J Palliat Med. 1998;1(3):221-230

25. Brumley R, Enguidanos S, Jamison P, Seitz R, Morgenstern $\mathrm{N}$, Saito $\mathrm{S}$, et al. Increased satisfaction with care and lower costs: results of a randomized trial of in-home palliative care. J Am Geriatr Soc.2007; 55(7): 993 \pm 1000 .

26. Rabow MW, Dibble SL, Pantilat SZ, McPhee SJ. The comprehensive care team: A controlled trial of outpatient palliative medicine consultation. Arch Intern Med. 2004; 164(1): $83 \pm 91$.

27. Wright AA, Keating NL, Balboni TA, et al. Place of death: correlations with quality of life of patients with cancer and predictors of bereaved caregivers' mental health. J Clin Oncol;2010; 28:4457-4464.

28. Campbell DE, Lynn J, Louis TA, Shugarman LR. Medicare program expenditures associated with hospice use. Annals of Internal Medicine 2004; 140(4):269-277.

\section{(i) (3)}

This work is licensed under a Creative Commons AttributionNon Commercial 4.0 International License. 